\title{
Using Parametric Multipoint Lods and Mods for Linkage Analysis Requires a Shift in Statistical Thinking
}

\author{
Susan E. Hodge ${ }^{\text {a }}$ Zeynep Baskurt ${ }^{\text {b }}$ Lisa J. Strug ${ }^{b, c}$ \\ a Division of Epidemiology, New York State Psychiatric Institute and Department of Psychiatry, Columbia College of \\ Physicians and Surgeons and Division of Statistical Genetics, Department of Biostatistics, Mailman School of Public \\ Health, Columbia University, New York, N.Y., USA; ${ }^{b}$ Child Health Evaluative Sciences, The Hospital for Sick Children, \\ and ' Dalla Lana School of Public Health, University of Toronto, Toronto, Ont., Canada
}

\section{Key Words}

Evidential paradigm • Likelihood • Parametric linkage •

Complex disease

\begin{abstract}
Multipoint (MP) linkage analysis represents a valuable tool for whole-genome studies but suffers from the disadvantage that its probability distribution is unknown and varies as a function of marker information and density, genetic model, number and structure of pedigrees, and the affection status distribution [Xing and Elston: Genet Epidemiol 2006; 30:447-458; Hodge et al.: Genet Epidemiol 2008;32:800815]. This implies that the MP significance criterion can differ for each marker and each dataset, and this fact makes planning and evaluation of MP linkage studies difficult. One way to circumvent this difficulty is to use simulations or permutation testing. Another approach is to use an alternative statistical paradigm to assess the statistical evidence for linkage, one that does not require computation of a $p$ value. Here we show how to use the evidential statistical paradigm for planning, conducting, and interpreting MP linkage studies when the disease model is known (lod analysis) or unknown (mod analysis). As a key feature, the evidential paradigm decouples uncertainty (i.e. error probabilities) from statistical evi-
\end{abstract}

dence. In the planning stage, the user calculates error probabilities, as functions of one's design choices (sample size, choice of alternative hypothesis, choice of likelihood ratio (LR) criterion $k$ ) in order to ensure a reliable study design. In the data analysis stage one no longer pays attention to those error probabilities. In this stage, one calculates the LR for two simple hypotheses (i.e. trait locus is unlinked vs. trait locus is located at a particular position) as a function of the parameter of interest (position). The LR directly measures the strength of evidence for linkage in a given data set and remains completely divorced from the error probabilities calculated in the planning stage. An important consequence of this procedure is that one can use the same criterion $k$ for all analyses. This contrasts with the situation described above, in which the value one uses to conclude significance may differ for each marker and each dataset in order to accommodate a fixed test size, $\alpha$. In this study we accomplish two goals that lead to a general algorithm for conducting evidential MP linkage studies. (1) We provide two theoretical results that translate into guidelines for investigators conducting evidential MP linkage: (a) Comparing mods to lods, error rates (including probabilities of weak evidence) are generally higher for mods when the null hypothesis is true, but lower for mods in the presence of true linkage. Royall [J Am Stat Assoc 2000;95:760-780] has shown that errors based on

\section{KARGER \\ Fax +4161306 1234 \\ E-Mail karger@karger.ch}

() 2011 S. Karger AG, Basel

www.karger.com
Accessible online at: www.karger.com/hhe
Lisa J. Strug, PhD

The Hospital for Sick Children

555 University Avenue

Toronto, ON M5G 1X8 (Canada)

Tel. +1 4168137654 ext. 1762, E-Mail lisa.strug@ utoronto.ca 
lods are bounded and generally small. Therefore when the true disease model is unknown and one chooses to use mods, one needs to control misleading evidence rates only under the null hypothesis; (b) for any given pair of contiguous marker loci, error rates under the null are greatest at the midpoint between the markers spaced furthest apart, which provides an obvious simple alternative hypothesis to specify for planning MP linkage studies. (2) We demonstrate through extensive simulation that this evidential approach can yield low error rates under the null and alternative hypotheses for both lods and mods, despite the fact that mod scores are not true LRs. Using these results we provide a coherent approach to implement a MP linkage study using the evidential paradigm.

Copyright $\odot 2011$ S. Karger AG, Basel

\section{Introduction}

Linkage analysis can provide an important tool for gene mapping on its own, and can provide data to guide prioritization of potential causal sequence variants identified as a part of next-generation sequence studies. It is commonplace for linkage studies to compute lod scores or mod scores (lod scores maximized over unknown disease model parameters), and to declare significant linkage when the observed lod/mod score exceeds some critical value deemed significant, where that critical value is determined by frequentist paradigm arguments. (Other published alternatives do exist, e.g. the posterior probability of linkage (e.g. Vieland [1]) and the false discovery rate (e.g. Devlin et al. [2])). That is, a given, fixed type I error level (test size $\alpha$ ) determines the critical value at which one declares significant linkage evidence, and this critical value is calculated from the probability distribution of the lod score.

In multipoint (MP) linkage analysis, one computes the MP lod/mod score based on two simple hypotheses for the location parameter (i.e. trait locus is unlinked vs. trait locus is located at a particular position), and for this scenario, standard likelihood ratio (LR) testing theory does not apply. The distribution of the test statistic (lod or mod) under the null hypothesis does not have a standard form. Rather, this distribution varies as a function of marker information and density, genetic model, number and structure of pedigrees, and the affection status distribution $[3,4]$. Consequently, the critical value at which the MP lod or mod score can be declared significant can vary by dataset and even by marker. Xing and Elston [3] suggest that, as a consequence, one should take care in

Using Parametric MP Lods and Mods for

LA Requires a Shift in Statistical Thinking interpreting MP parametric lod scores. However, Hodge et al. [4] point out that despite the unknown and varying critical value, the probability of obtaining strong evidence favoring linkage from the MP lod score when there is no linkage (referred to as $M_{0}$ ), converges to zero with increasing information. This behavior was already known for lod scores (proven by Royall [5]) and we demonstrated this via simulation for mods previously [4]. This implies that MP lod scores provide reliable linkage evidence on their own, irrespective of their corresponding frequentist critical value. Interpreting the statistical evidence for linkage directly from the LR or lod score is, in fact, an implementation of the evidential statistical paradigm.

The evidential paradigm [5-7] provides an alternative way to assess statistical evidence in a given dataset. In this paradigm, one bases inference solely on pure likelihood methods rather than using alternative measures of statistical evidence such as $p$ values or Bayes factors. One uses the LR, calculated from two simple hypotheses for the parameter of interest, as the measure of evidence. One also calculates error probabilities, in the forms of probabilities of misleading evidence and probabilities of weak (inconclusive) evidence, to provide measures of the operating characteristics of the paradigm under different scenarios. These probabilities ensure that interpreting linkage evidence directly from the LR has a low probability of leading to incorrect conclusions about linkage. However, these error rates play no role in the subsequent interpretation of the statistical evidence for linkage, which is based solely on the value of the observed LR for two simple hypotheses. We review the paradigm more fully in Section 2.

Evidential methodology has been developed for diverse applications including, for example, bioequivalence trials [8], nonparametric approaches [9], health economics [10], robust regression [11], two-point linkage analysis with known disease model [12], sample size estimation [13], and genetic association analysis [14], as well as for dealing with the multiple testing paradox [15]. More complete details can be found in those papers. Here we extend this suite of evidential methodology to MP linkage analysis, under both known and unknown disease models, allowing investigators to conduct MP linkage analysis evidentially.

The evidential paradigm draws the important distinction between uncertainty and statistical evidence [7]: The LR measures the strength of statistical evidence, and this measure is mathematically and conceptually distinct from the frequency with which misleading evidence could occur (error probabilities). As a consequence, the 
Box 1. Algorithm to conduct multipoint linkage analysis using the evidential paradigm

\section{(A) Study Design}

Among all adjacent pairs of markers, choose the pair whose two loci are furthest apart. Call the distance between them $d$.

- Simulate $N$ replicates of a dataset consisting of pedigrees with structure and disease distribution as in one's dataset, and two markers separated by distance $d$.

- Simulate both with linkage (disease locus located halfway between the two markers) and without linkage (disease locus located elsewhere) (Sec. 3.2).

- If genetic model of trait is known, use that to calculate error probabilities based on lods; if not, calculate error probabilities based on mod scores.

- Calculate $M_{0}$ and $W_{0}$ from the unlinked simulations (i.e. no linkage), and $W_{1}$ from the linked simulations, for chosen value of $k$ (e.g., $k=32$ ) (Sec. 3.1)

Ensure $M_{0}$ is small (e.g., $<0.05$ ), so that one has low probability of being misled by one's data.

Minimize $W_{0}$ and $W_{1}$ as much as possible.
(B) Study Analysis

Use multipoint linkage software to calculate genome-wide mod scores (or lod scores if trait model is known).

- Where $\bmod (\mathrm{lod}) \geq k$, interpret this as strong evidence favoring linkage at that location.

- e.g., if $k=32$, it is 32 times more likely than not that the trait locus is located here.

Compute the support interval about the maximum (see Sec. 2.1) to obtain a set of genomic locations consistent with linkage.

- This provides a set of locations across the genome whose linkage evidence is not substantially less than the evidence for linkage at the maximum.

Summarize regions of weak evidence - where LR is between $k$ and $1 / k$ - where one can neither conclude nor rule out linkage. evidential paradigm has the advantage of interpreting the evidence in the observed data set, as opposed to interpreting statistical evidence in terms of repetitions of the same experiment.

Another advantage of the evidential paradigm is that one can determine, after performing the analysis, whether one actually does have inconclusive ('weak') evidence or not. This contrasts with a power analysis in the frequentist approach, wherein one has only a probability ahead of time of being unable to reject the null hypothesis. Moreover, a power analysis does not distinguish between evidence that is truly inconclusive and evidence that actually strongly supports the null, as an evidential analysis does.

In addition to these and the other usual advantages of the evidential over the frequentist paradigm $[6,16]$, in MP linkage analysis the investigator can specify a common criterion for all markers and all studies. In contrast, under the frequentist paradigm, it is not straightforward to determine the required critical value to ensure a size $\alpha$ test corresponding to the MP lod/mod score, since the distribution of the MP lod is, in general, unknown [3-5]. Rather, once the $\alpha$ level is fixed at some value, say 0.05 , then the criterion for linkage (e.g. 3.3) needs to be different, depending on the distribution of the MP lod/mod. In contrast, with the evidential paradigm, the user specifies a cutoff, choosing a criterion that corresponds to the desired level of 'strength of evidence'.

In Section 2 we review salient aspects of the evidential paradigm. In Section 3 we develop two useful guidelines for investigators applying this paradigm. Then in Sections 4 (Methods) and 5 (Results) we present numerical results from our extensive simulations that, when combined with results from [4], illustrate how MP lod and mod scores can provide reliable linkage evidence in the absence of a corresponding frequentist critical value. Finally, in the Discussion (Section 6) we tie all this together to outline a coherent evidential approach to conducting a MP linkage study. Box 1 summarizes the overall algorithm involved in conducting a MP linkage analysis using the evidential paradigm.

\section{Review of the Evidential Paradigm and Previous Work}

Royall [6] and Blume [7] introduced the evidential paradigm, and the methodology for two-point linkage analysis for known disease models was developed by Strug and Hodge [12]. Since evaluating the MP lod and mod scores over the whole genome is analogous to evaluating two-point lod and mod scores over the range of possible 
recombination fractions, the evidential paradigm is straightforward to apply to MP linkage analysis. Here we briefly summarize.

\subsection{LR and the Criterion for Strong Evidence}

To formulate the LR, one puts the null hypothesis $H_{0}$ of 'no linkage' in the denominator. For the numerator, one inserts a simple alternative hypothesis, $H_{1}$ (also see Sec. 3.2). Once the data have been observed, the investigator calculates and reports the $L R$ as a function of the parameter of interest (in MP linkage analysis, that parameter is the location in the genome). The investigator then interprets each ratio as the strength of the evidence favoring various alternatively hypothesized parameter values over the null hypothesized value of no linkage.

One chooses a value $k$ as the criterion for 'strong evidence', such that if a $L R$ is greater than or equal to $k$, this is interpreted as strong evidence in favor of the hypothesis in the numerator, whereas if the $L R$ is less than or equal to $1 / k$, that is strong evidence for the hypothesis in the denominator. ${ }^{1}$ Benchmarks for $k$ have been suggested and justified in the literature $[6,7,16]$. Some reasonable values of $k$ are 32 and 100, whereas in the linkage literature, classically a value of $k=1,000$ (corresponds to a lod score of 3.0) has been used.

Additionally, $1 / k$ support intervals are calculated to give a set of parameter values that are consistent with the data, since the MLE is not better supported over these parameter values by a factor of $\mathrm{k}$ or more; these are analogous to confidence intervals yet have a different interpretation $[6,7,9,16,17]$. For lods and mods, one works with the $\log _{10} L R$, so one uses $c$, defined as $c \equiv \log _{10} k$, as the corresponding criterion. If the lod or mod falls between $c$ and $-c$, that outcome is interpreted as 'weak evidence', i.e. evidence that is not strong enough to favor either of the two hypotheses.

\subsection{The Error Probabilities}

Prior to data analysis, error probabilities are calculated from two simple hypotheses, the one representing 'no linkage', and the other a single simple alternative (e.g. a position across the genome for MP linkage analysis, or a specific value of the recombination fraction for two-point linkage analysis). The simple alternative is chosen for planning purposes only and has no effect on the investigator's

\footnotetext{
1 The two criteria need not be symmetric. One can specify one value $k>1$ for the level of evidence required to accept $\mathrm{H}_{1}$, but use $1 / \mathrm{l}$ as the criterion for accepting $\mathrm{H}_{0}$, where another value $\mathrm{l}>1$ can be either greater than or less than $k$ ). See Strug and Hodge [12] for further discussion.
}

Using Parametric MP Lods and Mods for

LA Requires a Shift in Statistical Thinking ability to interpret the $L R$ at other simple alternatives once the data have been observed, as we explain further below.

If the null hypothesis is true, then a small $L R$ leads to the correct conclusion, whereas a large one gives misleading information. The first outcome is referred to as 'strong' evidence and the second one as 'misleading' evidence. We denote their respective probabilities as follows:

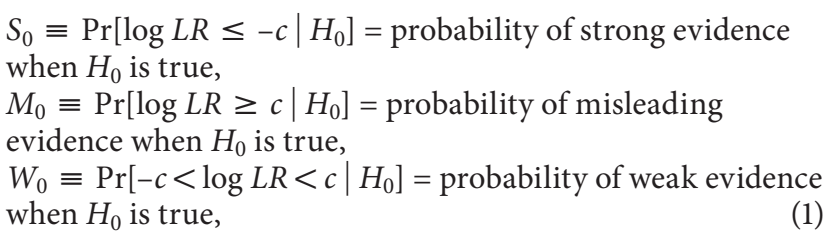

where $W_{0}$ represents the third outcome, in which $\log L R$ falls between the two criteria. If the alternative hypothesis is true, a large LR leads to the correct conclusion, so we define:

$S_{1} \equiv \operatorname{Pr}\left[\log L R \geq c \mid H_{1}\right]=$ probability of strong evidence when $H_{1}$ is true,

$M_{1} \equiv \operatorname{Pr}\left[\log L R \leq-c \mid H_{1}\right]=$ probability of misleading evidence when $H_{1}$ is true,

$W_{1} \equiv \operatorname{Pr}\left[-c<\log L R<c \mid H_{1}\right]=$ probability of weak evidence when $H_{1}$ is true.

Note that it follows from (1) and (2) that

$S_{i}+W_{i}+M_{i}=1$, for $i=0,1$.

From (1) and (2), the reader can see that $M_{0}$ is analogous to type I error $(\alpha)$, and $S_{1}$ is analogous to power, in the frequentist paradigm. However, $M_{0}$ plays a different role than type I error: Instead of fixing $\alpha$, and then seeing what cutoff value that corresponds to, the investigator fixes the cutoff value $k$ (or $c$ on the $\log _{10}$ scale), then determines what the error levels are. The investigator is concerned with the corresponding $M_{0}$ (the evidential analog to type I error) only in the planning phase. Moreover, the investigator is less concerned with the actual value of this error probability and simply wants to ensure that it remains 'small' across the genome (i.e. less than some value, such as 0.05) [7]. This guarantees that interpreting strong evidence vis-à-vis the LR for two simple hypotheses has a low probability of leading one to draw incorrect conclusions about linkage evidence. Royall [5] has derived bounds that ensure this for lod scores. Here we extend this reasoning to MP mod scores, and we also investigate what happens under the alternative hypothesis (i.e. when the true state of nature is linkage).

The corresponding $M_{0}$ plays no role in the interpretation of the evidence strength, or in what critical values are required to interpret a given lod or mod score as representing strong linkage evidence or lack thereof; the error 
probabilities merely provide an assurance that the study design is reliable. This is because in the evidential paradigm, error probabilities and evidence strength are independent concepts $[7,12]$. That is, their probability values do not affect the strength of the statistical evidence in the observed data, nor do they affect the probability that the observed evidence is misleading.

Royall [5] has shown that the probability of misleading evidence is bounded asymptotically for quite general one-parameter models (e.g. MP lod scores), for multi-parameter fixed-dimensional models, and even for profile likelihoods. In large samples, the type I error analog, $M_{0}$, cannot exceed $\Phi(-\sqrt{2 \ln k})$ for any sample size or any alternatively hypothesized parameter value, where $\Phi$ is the standard normal cumulative density function (this result also holds for $M_{1}$ ). Therefore, for MP lod scores in large samples we can be confident that both $M_{0}$ and $M_{1}$ will remain small, and thus one is able to interpret the statistical evidence for linkage directly from the MP lod score, irrespective of a frequentist critical value. MP mod scores, on the other hand, are not LRs or profile LRs since they maximize the ratio over the unknown trait parameters in numerator and denominator simultaneously ${ }^{2}$. Here we will evaluate the evidential error rates for MP mod scores via simulation, in order to ensure that MP mod scores are embodied by the operational characteristics fundamental to the evidential paradigm: (1) small $M_{0}(n, k)$ and $M_{1}(n, k)$ and (2) decreasing $W_{0}(n, k)$ and $W_{1}(n, k)$ with increasing sample size. These results will justify use of the evidential paradigm in MP linkage studies when the disease model is unknown and requires maximization over multiple trait parameters.

\subsection{Our Previous Work}

In Strug and Hodge [12] we applied the evidential paradigm to classical two-point linkage analysis, under simple generating models (GMs). We illustrated applications of the evidential paradigm in fully informative gametes, in double backcross sibling pairs, and in nuclear families, analyzing single-gene traits for which the disease model is known but reduced penetrance may be incorrectly specified. We combined analytical studies and simulations to develop the operational characteristics of the evidential paradigm as applied to linkage analysis, illustrating the resulting low rates of incorrect conclusions (i.e.

\footnotetext{
2 A profile LR is constructed by maximizing separate profile likelihood functions $\left(\max _{\phi} \mathrm{L}(\phi)\right)$ in the numerator and denominator of the ratio, where $\phi$ is the unknown trait model. In contrast, a mod score is defined as $\max _{\phi} \log 10 \mathrm{LR}(\phi)$, i.e. the unknown trait model parameters are maximized over the whole ratio.
}

small values of $M_{0}$ and $\left.M_{1}\right)$. We showed that, for reasonable values of $k$ and of the recombination fraction specified under $H_{1}$ (i.e. $\theta_{1}$ ), $M_{0}$ and $M_{1}$ were naturally very low, and $W_{0}$ and $W_{1}$ could be well controlled with sample size during the planning phase.

As part of the work in Hodge et al. [4] we presented some results for $M_{0}$ for MP lods and mods for two simple hypotheses (as computed in the evidential paradigm). In extensive simulations we confirmed that within the limits of simulation, not only were the $M_{0}$ very low, but, even more importantly, they decreased with increasing sample size for both MP lods and MP mods. (As mentioned in the Introduction, the lod results for two simple hypotheses follow directly from Royall [5], but the mod results required investigation by simulation.)

For example, we generated nuclear families under a dominant or recessive model with reduced penetrance, and analyzed them under the same model; i.e. the analysis model (AM) was the same as the GM for each analysis (lod analyses). Even when we used the low lod cutoff value of $c=0.9$ (corresponds to $k=8$ ), values of $M_{0}$ quickly dropped from very small (between 0.001 and 0.022 ) to essentially zero as dataset size increased from 10 to 30 families. For the corresponding mod analyses, using the same cutoff value, $M_{0}$ values were of course higher (though still below 0.04 even for datasets as small as 10 families) and required dataset sizes of 75 families before they approached zero; however, when we used a cutoff of $c=1.5$ $(k=32), M_{0}$ values were near zero by the time dataset size reached 50 families. For detailed numerical results see table 1 and figure 1 in Hodge et al. [4].

In the current study we continue with the situation of two simple hypotheses, and we extend these results to $W_{0}$, as well as studying the behavior of the error probabilities for MP lods and mods under the alternative hypothesis of linkage, in order to ultimately present a coherent algorithm to implement MP linkage analysis using the evidential paradigm (Box 1).

\section{Two Guidelines for Conducting Evidential MP Linkage Analysis}

In order to conduct an MP linkage study evidentially, i.e. to calculate a lod/mod score and interpret the strength of the linkage evidence from that lod/mod value itself, one needs to know that the probability of errors is small for a given criterion, $k$, sample size and parameter vector. For lods we know the behavior of the error rates from Royall [5]. For mods, simulation is required in order to explore 
the behavior of these error probabilities. When we estimate the size of these error rates, two questions arise: (1) When is it sufficient to base error rate estimates on lods (i.e. rely on large sample results) rather than calculate the mod errors (Section 3.1)? (2) What parameter value should be used for the simple alternative hypothesis, to calculate errors (Section 3.2)? Box 1(A) summarizes the roles of these two guidelines in an evidential MP linkage study, while Box 1(B) provides the analysis algorithm.

\subsection{Relative Magnitudes of Errors for Lods and Mods}

Obviously, if one uses the same cutoff value for both lods and mods, the mod error rates will be higher than lod error rates if the true state of nature is 'no linkage,' and lower if the true state of nature is 'linkage'. Thus, both $M_{0}$ and $S_{1}$ will be higher for mods than for lods.

It is less clear what happens to the $W_{i}$, the probabilities of weak evidence. It turns out that under some reasonable assumptions, $W_{0}$ is greater for mods, whereas $W_{1}$ is greater for lods; thus the $W_{i}$ parallel the behavior of the $M_{i}$, not of the $S_{\mathrm{i}}$. Table 1 summarizes these relationships, and our simulations (described below) demonstrate the patterns. Section 2 of the Appendix gives proofs for the behavior of the $\mathrm{M}$ and $\mathrm{S}$, Section 3 gives supporting arguments for the behavior of the W.

In summary, since under the alternative, mod error rates are less than lod error rates, and since the $M_{1}$ are bounded for lods [5], we can be assured that $M_{1}$ calculated from mods are small. Therefore, assuming MP mod scores under the alternative behave reliably according to the operational characteristics of the evidential paradigm (i.e. have small $M_{1}(n, k)$ and decreasing $W_{1}(n, k)$ with increasing sample size), one needs to calculate only $M_{0}$ based on mods in order to ensure low misleading error rates for a given study. For a well-designed study, however, one should be aware of the study's corresponding probabilities of weak evidence, $\mathrm{W}_{0}$ and $\mathrm{W}_{1}$, and sample size should be chosen as best as possible to minimize these probabilities.

\subsection{Choosing the Alternative Hypothesis for Planning Purposes}

In order to calculate the required planning probabilities $\mathrm{M}_{0}, \mathrm{~W}_{0}$, and $\mathrm{W}_{1}$, the user must specify not only the null hypothesis of no linkage, but also a simple alternative hypothesis. This simple alternative should be the most conservative choice such that the corresponding probability for this simple alternative serves as an upper bound for any pair of markers in the genome scan. It is easy to misunderstand the role of this alternative hypothesis and to
Table 1. Relative magnitudes of S, W, and $\mathrm{M}$ for lods versus mods

\begin{tabular}{ll}
\hline When $H_{0}$ is true & When $H_{1}$ is true \\
\hline$S_{0} \bmod \leq S_{0}$ lod & $S_{1} \bmod \geq S_{1}$ lod \\
$W_{0} \bmod \geq W_{0}$ lod & $W_{1} \bmod \leq W_{1} \operatorname{lod}$ \\
$M_{0} \bmod \geq M_{0} \operatorname{lod}$ & $M_{1} \bmod \leq M_{1} \operatorname{lod}$
\end{tabular}

Conclusions for $S$ and $M$ are proven rigorously (Appendix, Section 2). Conclusions for $W$ are based on approximate arguments (Appendix, Section 3) and are borne out in the simulations for $W_{0}$ and $W_{1}$ (tables 4 and 6, respectively).

think that specifying it limits one's analysis or one's abilities to draw conclusions from one's data. However, that is not the case. The alternative hypothesis plays a role only in planning the study and plays no role in data analysis.

To understand this, consider that between any two marker loci A and B, the greatest probability of misleading evidence when there is no linkage (i.e. the maximum $M_{0}$ ) will occur at the midpoint between the two loci. This is true since at positions closer to either locus, there is more evidence against linkage with that locus. Table 2 illustrates this phenomenon for two loci separated by $10 \mathrm{cM}$. The table shows values of $M_{0}$ (exact, not simulated), calculated at distances of $0.1,1.0,2.0,3.0,4.0$, and $5.0 \mathrm{cM}$ from the first locus, and illustrates how the maximum $M_{0}$ always occurs in the middle (calculations as in Hodge et al. [4]). Thus, one can safely choose the midpoint between two markers to represent the alternative simple hypothesis. This provides a conservative estimate of the error rate under $\mathrm{H}_{0}$, since for any other alternative that error rate would be smaller. Likewise, for any other alternative, $\mathrm{W}_{0}$ is smaller. This implies that the largest sample size estimate also comes from the choice of the alternative at the midpoint.

Further, among all pairs of contiguous marker loci, one can pick that pair whose two loci are spaced furthest apart. The results for that pair will then be conservatively applicable to all other pairs. (Also see last paragraph of Section 5, Simulation Results, as well as Box 1.)

\section{Simulation Methods}

\subsection{Generating Model}

Here we use the same simulation models as in Hodge et al. [4]. Briefly, we simulated two marker loci, $A$ and $B$, separated by a distance of $10 \mathrm{cM}$. The trait was due to either a dominant or recessive model with reduced penetrance. Let $T$ represent the trait allele and $t$, the wild type. For the dominant GMs, the frequency of $T$ was 0.01 , and the penetrance vector for genotypes $(T T, T t, t t)$ 
Table 2. Values of $M_{0}$ at positions between markers A and B, for cutoff value $c=0.9$, for different numbers (n) of fully informative gametes

\begin{tabular}{|c|c|c|c|c|c|c|}
\hline \multirow[t]{2}{*}{$\mathrm{n}$} & \multicolumn{6}{|c|}{ Distance from locus A, cM } \\
\hline & 0.1 & 1.0 & 2.0 & 3.0 & 4.0 & 5.0 \\
\hline 10 & 0.0019 & 0.0022 & 0.0023 & 0.0023 & 0.0023 & 0.0023 \\
\hline 12 & 0.0005 & 0.0006 & 0.0007 & 0.0016 & 0.0016 & 0.0016 \\
\hline 14 & 0.0001 & 0.0010 & 0.0010 & 0.0013 & 0.0016 & 0.0017 \\
\hline 16 & 0.0000 & 0.0003 & 0.0006 & 0.0007 & 0.0008 & 0.0008 \\
\hline 18 & 0.0001 & 0.0002 & 0.0002 & 0.0003 & 0.0003 & 0.0003 \\
\hline 20 & 0.0000 & 0.0001 & 0.0001 & 0.0001 & 0.0001 & 0.0001 \\
\hline 25 & 0.0000 & 0.0000 & 0.0000 & 0.0000 & 0.0000 & 0.0000 \\
\hline
\end{tabular}

Locus A and B are separated by $10.0 \mathrm{cM}$.

Numbers in the table result from exact calculations, not simulations.

Table 3. Values of $M_{1}$ for lods and mods in 1,000 simulated datasets, as a function of dataset size, for cutoff value $c=0.9(k=8)$, evaluated at a position halfway between two markers separated by $10 \mathrm{cM}$

\begin{tabular}{lllllll}
\hline GM & \multicolumn{5}{l}{ Number of families } \\
\cline { 2 - 7 } & 10 & 20 & 30 & 40 & 50 & 75 \\
\hline a Lods & & & & & & \\
D20 & 0.009 & 0.004 & 0.001 & 0.002 & 0.000 & 0.000 \\
D50 & 0.006 & 0.000 & 0.000 & 0.000 & 0.000 & 0.000 \\
D80 & 0.001 & 0.000 & 0.000 & 0.000 & 0.000 & 0.000 \\
R20 & 0.010 & 0.000 & 0.002 & 0.000 & 0.000 & 0.000 \\
R50 & 0.001 & 0.002 & 0.000 & 0.000 & 0.000 & 0.000 \\
R80 & 0.001 & 0.000 & 0.000 & 0.000 & 0.000 & 0.000 \\
\hline b Mods & & & & & & \\
D20 & 0.000 & 0.000 & 0.001 & 0.000 & 0.001 & 0.000 \\
D50 & 0.000 & 0.000 & 0.000 & 0.000 & 0.000 & 0.000 \\
D80 & 0.000 & 0.000 & 0.000 & 0.000 & 0.000 & 0.000 \\
R20 & 0.001 & 0.000 & 0.000 & 0.000 & 0.000 & 0.000 \\
R50 & 0.000 & 0.000 & 0.000 & 0.000 & 0.000 & 0.000 \\
R80 & 0.000 & 0.000 & 0.000 & 0.000 & 0.000 & 0.000 \\
\hline
\end{tabular}

was $(f, f, 0.001)$, where $f$ assumed the values $0.20,0.50$, or 0.80 (models D20, D50, and D80, respectively). For the recessive GMs, frequency of $T=0.14$, and penetrance vectors $=(f, 0.001,0.001)$, where $f$ assumed the same three values (models R20, R50, and R80, respectively).

For the linkage scenario, the trait locus was linked to both markers, either located halfway between the two marker loci (results shown in tables 3-5) or located at marker A (results not shown). Simulations were performed at the midpoint to start with, so as to correspond to the $\mathrm{H}_{0}$ simulations [4]. Results when the trait locus was located at one of the markers were very similar (see Results for details). For the nonlinkage scenarios, the trait locus was not linked to either marker.
We simulated four-child nuclear families with at least two affected children. Parents were typed and were fully informative except for phase (that is, both parents were heterozygous at both marker loci, with no marker alleles in common, but their phases were unknown), and parental affectedness status was known.

\subsection{Analysis Models}

We analyzed the simulated data assuming a number of AMs, among which the true model (the GM) was always included. Dominant AMs D10, D20, ..., D90 had penetrance vectors $(f, f$, 0.001 ) where now $f$ assumed values $0.1,0.2, \ldots, 0.9$, respectively; and similarly for recessive AMs R10 through R90. For each GM and each value of $n(n=10,20,30,40,50,75)$, we simulated $N=$ 1,000 datasets of $n$ families each. Each dataset was first analyzed via MP lods, i.e. where we assumed the correct GM in order to perform the analysis. Then each dataset was analyzed via MP mods; here we determined a max lod for each of the 18 different AMs, then determined the maximum of those 18 maxima. These AMs were chosen to be comparable to those used in the earlier papers $[3,4]$, and serve as an approximation to a true mod score, which would require an optimization procedure to calculate. Scores were calculated at only one position, located halfway between the two markers. We used cutoff values of $k=8$ and 32, or, equivalently on the common $\log$ scale, $c=0.9$ and 1.5 .

For each analysis, we tabulated the proportion of times, out of 1,000 , that the appropriate score (max lod or max mod) was less than or equal to $-c$ (this proportion represents $M_{1}$ ), fell between $-c$ and $c\left(W_{1}\right)$, or was greater than or equal to $c\left(S_{1}\right)$.

\section{Simulation Results}

$\mathrm{M}_{0}$ and $\mathrm{S}_{0}$ can be found in Table 1 of Hodge et al. [4]. Tables 3-5 here show the simulation results for $M_{1}, W_{1}$, $S_{1}$, respectively, when the trait locus is located halfway between markers $A$ and $B$. Table 6 shows results for $W_{0}$, i.e. when the trait locus is not linked to either marker. In 
Table 4. Values of $W_{1}$ for lods and mods in 1,000 simulated datasets, as a function of dataset size, for cutoff value $c=0.9(k=8)$ and and $c=1.5(k=32)$, evaluated at a position halfway between two markers separated by $10 \mathrm{cM}$

\begin{tabular}{|c|c|c|c|c|c|c|c|c|c|c|c|c|}
\hline \multirow[t]{2}{*}{ GM } & \multicolumn{12}{|c|}{ Number of families } \\
\hline & $\mathrm{k}=8$ & $\mathrm{k}=32$ & $\mathrm{k}=8$ & $\mathrm{k}=32$ & $\mathrm{k}=8$ & $\mathrm{k}=32$ & $\mathrm{k}=8$ & $\mathrm{k}=32$ & $\mathrm{k}=8$ & $\mathrm{k}=32$ & $\mathrm{k}=8$ & $\mathrm{k}=32$ \\
\hline \multicolumn{13}{|c|}{ a Lods } \\
\hline D20 & 0.269 & 0.576 & 0.072 & 0.163 & 0.017 & 0.044 & 0.009 & 0.024 & 0.005 & 0.013 & 0.000 & 0.000 \\
\hline D50 & 0.090 & 0.195 & 0.010 & 0.029 & 0.000 & 0.002 & 0.000 & 0.000 & 0.000 & 0.000 & 0.000 & 0.000 \\
\hline $\mathrm{R} 50$ & 0.032 & 0.088 & 0.004 & 0.008 & 0.000 & 0.000 & 0.000 & 0.000 & 0.000 & 0.000 & 0.000 & 0.000 \\
\hline R80 & 0.008 & 0.015 & 0.000 & 0.000 & 0.000 & 0.000 & 0.000 & 0.000 & 0.000 & 0.000 & 0.000 & 0.000 \\
\hline \multicolumn{13}{|c|}{ b Mods } \\
\hline D20 & 0.234 & 0.512 & 0.056 & 0.130 & 0.012 & 0.033 & 0.006 & 0.016 & 0.003 & 0.005 & 0.000 & 0.000 \\
\hline D50 & 0.061 & 0.143 & 0.004 & 0.013 & 0.000 & 0.001 & 0.000 & 0.000 & 0.000 & 0.000 & 0.000 & 0.000 \\
\hline
\end{tabular}

each table, part (a) shows the results for MP lods calculated under the correct model (the GM), whereas part (b) gives the results for MP lods maximized over the 18 models (MP mod) described above. We show results for $k=8$ and $k=32$, except in table $3\left(M_{1}\right)$, where we show only results for $k=8$, since almost all the $k=32$ results equaled zero.

For $M_{1}$ (table 3) we can see that for each of the six GMs considered, and for all sample sizes, we observe very few instances of misleading evidence, the worst case occurring under a recessive model with $20 \%$ penetrance, for $\mathrm{k}=8$ with 10 families $\left(M_{1}=0.010\right)$. Otherwise, we see that, with increasing information, these rates quickly approach 0 as $n$ increases, as would be predicted for lods based on Royall [5]. As expected (Appendix, Section 2), comparing table $3 \mathrm{a}$ to $\mathrm{b}$ confirms that $M_{1}$ is uniformly smaller after maximizing over the 18 AMs. Therefore, for the models considered here, which cover a broad grid of potential models, at relatively small sample sizes, $M_{1}$ is essentially negligible - even for low values of $k$.

Turning to table 4 and the probability of observing weak MP linkage signals when there is linkage we see that: $W_{1}$ increases with $k$, for a given GM and sample size; $W_{1}$ decreases with increasing sample size $n$, for a given $k$ and $\mathrm{GM}$; and $W_{1}$ decreases as penetrance increases, for a given $k$ and $n$. These results hold in both dominant and recessive models, for both lods and mods. Moreover, comparing individual cells from table $4 \mathrm{a}$ and $\mathrm{b}$ indicates that $W_{1}$ is smaller when one maximizes over all the 18 AMs rather than analyzing at just the true model, thus supporting the argument in Section 3 of the Appendix.

Table 5 shows the probabilities of strong evidence for lods (table 5a) and mods (table 5b). Since $M_{1}+W_{1}+S_{1}=$ 1 (eq. 3), it is not surprising to see that $S_{1}$ is smaller for larger $k$, is larger for larger $n$, and is larger for higher penetrances. Also, $S_{1}$ is always greater for mods than for lods when $\mathrm{H}_{1}$ is true (Appendix, Section 2).

Finally, table 6 gives the probabilities of weak evidence when there is no linkage $\left(\mathrm{H}_{0}\right)$. These results qualitatively mirror those for $W_{1}$, in both dominant and recessive models, for both lods and mods. That is, $W_{0}$ also increases with $k$, for a given GM and sample size; decreases with increasing sample size $n$, for a given $k$ and GM; and decreases as penetrance increases, for a given $k$ and $n$. Unlike $W_{1}, W_{0}$ is larger for mods than for lods, also supporting the argument in Section 3 of the Appendix.

Interestingly, the values for $\mathrm{W}_{0}$ are consistently larger than those for $\mathrm{W}_{1}$, as we see if we compare tables 4 and 6 , cell by cell. Yet misleading evidence probabilities $\left(\mathrm{M}_{\mathrm{i}}\right)$ remain very small under both $\mathrm{H}_{0}$ and $\mathrm{H}_{1}$. Table 7 illustrates what happens to $W_{0}$ and $W_{1}$ as we move from lods to mods, for some typical examples. The table gives $\mathrm{M}, \mathrm{W}$, and $S$ for two GMs (one dominant, one recessive, both with $50 \%$ penetrance) and datasets of 20 families, using $k=32$ as the criterion. Under $\mathrm{H}_{1}$ (part b of table 7), mov- 
Table 5. Values of $S_{1}$ for lods and mods in 1,000 simulated datasets, as a function of dataset size, for cutoff value $c=0.9(k=8)$ and $c=$ $1.5(k=32)$, evaluated at a position halfway between two markers separated by $10 \mathrm{cM}$

\begin{tabular}{|c|c|c|c|c|c|c|c|c|c|c|c|c|}
\hline \multirow[t]{2}{*}{ GM } & \multicolumn{12}{|c|}{ Number of families } \\
\hline & $\mathrm{k}=8$ & $\mathrm{k}=32$ & $\mathrm{k}=8$ & $\mathrm{k}=32$ & $\mathrm{k}=8$ & $\mathrm{k}=32$ & $\mathrm{k}=8$ & $\mathrm{k}=32$ & $\mathrm{k}=8$ & $\mathrm{k}=32$ & $\mathrm{k}=8$ & $\mathrm{k}=32$ \\
\hline \multicolumn{13}{|c|}{ a Lods } \\
\hline D20 & 0.722 & 0.424 & 0.924 & 0.836 & 0.982 & 0.955 & 0.989 & 0.975 & 0.995 & 0.987 & 1.000 & 1.000 \\
\hline D50 & 0.904 & 0.805 & 0.990 & 0.971 & 1.000 & 0.998 & 1.000 & 1.000 & 1.000 & 1.000 & 1.000 & 1.000 \\
\hline $\mathrm{R} 50$ & 0.967 & 0.912 & 0.994 & 0.992 & 1.000 & 1.000 & 1.000 & 1.000 & 1.000 & 1.000 & 1.000 & 1.000 \\
\hline $\mathrm{R} 80$ & 0.991 & 0.984 & 1.000 & 1.000 & 1.000 & 1.000 & 1.000 & 1.000 & 1.000 & 1.000 & 1.000 & 1.000 \\
\hline \multicolumn{13}{|c|}{ b Mods } \\
\hline D20 & 0.766 & 0.488 & 0.944 & 0.870 & 0.987 & 0.967 & 0.994 & 0.984 & 0.997 & 0.995 & 1.000 & 1.000 \\
\hline D50 & 0.939 & 0.857 & 0.996 & 0.987 & 1.000 & 0.999 & 1.000 & 1.000 & 1.000 & 1.000 & 1.000 & 1.000 \\
\hline
\end{tabular}

Table 6. Values of $W_{0}$ for lods and mods in 1,000 simulated datasets, as a function of dataset size, for cutoff value $c=0.9(k=8)$ and and $c=1.5(k=32)$, evaluated at a position halfway between two markers separated by $10 \mathrm{cM}$

\begin{tabular}{|c|c|c|c|c|c|c|c|c|c|c|c|c|}
\hline \multirow[t]{2}{*}{ GM } & \multicolumn{12}{|c|}{ Number of families } \\
\hline & $\mathrm{k}=8$ & $\mathrm{k}=32$ & $\mathrm{k}=8$ & $\mathrm{k}=32$ & $\mathrm{k}=8$ & $\mathrm{k}=32$ & $\mathrm{k}=8$ & $\mathrm{k}=32$ & $\mathrm{k}=8$ & $\mathrm{k}=32$ & $\mathrm{k}=8$ & $\mathrm{k}=32$ \\
\hline \multicolumn{13}{|c|}{ a Lods } \\
\hline D20 & 0.509 & 0.709 & 0.287 & 0.447 & 0.157 & 0.280 & 0.095 & 0.165 & 0.043 & 0.097 & 0.016 & 0.028 \\
\hline D50 & 0.294 & 0.459 & 0.106 & 0.179 & 0.035 & 0.053 & 0.013 & 0.023 & 0.008 & 0.016 & 0.001 & 0.002 \\
\hline R50 & 0.175 & 0.291 & 0.025 & 0.061 & 0.009 & 0.022 & 0.004 & 0.005 & 0.000 & 0.001 & 0.000 & 0.000 \\
\hline R80 & 0.066 & 0.122 & 0.005 & 0.009 & 0.001 & 0.003 & 0.000 & 0.000 & 0.000 & 0.000 & 0.000 & 0.000 \\
\hline \multicolumn{13}{|c|}{ b Mods } \\
\hline D20 & 0.857 & 0.973 & 0.686 & 0.906 & 0.497 & 0.723 & 0.409 & 0.622 & 0.325 & 0.521 & 0.179 & 0.321 \\
\hline D50 & 0.660 & 0.879 & 0.405 & 0.615 & 0.220 & 0.396 & 0.129 & 0.252 & 0.091 & 0.144 & 0.021 & 0.043 \\
\hline
\end{tabular}

ing from lods to mods has only a small effect on the probabilities of strong evidence, $S_{1}$ (already high), and weak evidence, $\mathrm{W}_{1}$ (already low). In contrast, under $\mathrm{H}_{0}$ (table $7 \mathrm{a}$ ), the probability of strong evidence, $\mathrm{S}_{0}$, goes from reasonably high ( 0.82 for the dominant example, 0.94 for the recessive one) to much lower (0.37 dominant, 0.035 recessive). Yet almost all of that shift shows up in $\mathrm{W}_{0}$, the probability of weak evidence, not in misleading evidence, which rises only slightly. In other words, although $\mathrm{M}_{0}$ does increase in mods as compared to lods (Appendix, Section 2), even for mods it remains very small, as we had shown in our earlier work [4]. 
We also examined what happens under $\mathrm{H}_{1}$ when the trait locus is located at one of the two marker loci. The results (not shown) for these runs are qualitatively similar to those where the trait locus is halfway between the two markers, but are slightly more favorable, in that the probabilities of weak evidence $\left(W_{1}\right)$ are slightly smaller, and the probabilities of strong evidence $\left(S_{1}\right)$ slightly greater. Therefore, the results shown in the tables represent the worst-case scenarios.

\section{Discussion}

\subsection{Summary}

In this study we have investigated via simulation the operational characteristics of the evidential paradigm when applied to MP linkage analysis for both lod and mod analysis. We have demonstrated that the operational characteristics of the evidential paradigm we observed in Strug and Hodge [12] continue to hold for MP mod scores despite being non-standard evidence functions, allowing us to extend the paradigm to MP linkage analysis with unknown disease models.

Here we have provided the theoretical results required to guide investigators on how to calculate the error probabilities of the evidential paradigm, described in Section 3 and summarized in Box 1. We have also performed extensive simulations, which indicate that the evidential approach can yield low error rates under the null and alternative hypotheses for both lods and mods (tables 3-6).

\subsection{Favorable Operating Characteristics of the Evidential Paradigm}

The evidential paradigm is straightforward to apply to MP linkage studies, requiring only that MP lods or mods be calculated. The strength of the linkage evidence can then be interpreted directly from the observed value of the lod/mod score.

We have seen that although the probability of observing misleading evidence is, in general, quite low, the corresponding probabilities of weak evidence can be large. We reiterate that, just as in the two-point linkage case [12], during the planning stage investigators should focus more on minimizing weak signals, than on minimizing the misleading evidence probabilities. If the probability of weak evidence is too high, one can lower it either by increasing sample size or by choosing a lower value of $k$. Increasing sample size is the ideal solution but is not always possible. However, it may be feasible to lower $k$. Investigators tend to focus on reducing type I error proba-
Table 7. Illustrative values of $M_{i}, W_{i}$, and $S_{i}$ for one dominant and one recessive model, in datasets of size $\mathrm{n}=20$, using $k=32$

a Under $\mathrm{H}_{0}$

\begin{tabular}{lllllllll}
\hline & \multicolumn{3}{c}{$\mathrm{GM}=\mathrm{D} 50$} & & & \multicolumn{2}{l}{$\mathrm{GM}=\mathrm{R} 50$} \\
\cline { 2 - 3 } \cline { 7 - 8 } & $M_{0}$ & $W_{0}$ & $S_{0}$ & & $M_{0}$ & $W_{0}$ & $S_{0}$ \\
\hline Lod & 0.004 & 0.179 & 0.817 & & 0.001 & 0.177 & 0.938 \\
Mod & 0.016 & 0.615 & 0.369 & & 0.009 & 0.956 & 0.035 \\
\hline
\end{tabular}

b Under $\mathrm{H}_{1}$

\begin{tabular}{lllllllll}
\hline & \multicolumn{3}{l}{$\mathrm{GM}=\mathrm{D} 50$} & & \multicolumn{3}{l}{$\mathrm{GM}=\mathrm{R} 50$} \\
\cline { 7 - 8 } & $M_{1}$ & $W_{1}$ & $S_{1}$ & & $M_{1}$ & $W_{1}$ & $S_{1}$ \\
\hline Lod & 0.000 & 0.029 & 0.971 & & 0.000 & 0.008 & 0.992 \\
Mod & 0.000 & 0.013 & 0.987 & & 0.000 & 0.001 & 0.999 \\
\hline
\end{tabular}

bilities in the frequentist paradigm, which in turn corresponds to larger required critical values analogous to the role of $k$. In the evidential paradigm, raising $k$ has only a minor effect on lowering the probabilities of misleading evidence, but a larger effect on raising the probabilities of weak evidence [13] (also see tables 3-5 in this paper). Instead, investigators should focus on minimizing weak evidence probabilities, and for that purpose, one should not increase $k$ beyond what is compelling $[6,16]$. This concept of explicitly minimizing the probability of weak evidence represents a novel contribution of the evidential paradigm [18].

\subsection{Advantage of Single Criterion}

We have shown that, in contrast to frequentist analysis of MP linkage, with the evidential paradigm one can use a common linkage criterion for MP studies, presuming an investigator has established that the evidential error probability of misleading evidence for a proposed MP linkage study is small. Using the frequentist paradigm, if one requires type I error control of, for example, $5 \%$, then the linkage criterion for significance would be different at each marker and across every study.

\subsection{Meaning of Error Probabilities in the Evidential Paradigm}

We return to the subject of what the error $M_{0}$ actually means. We have referred to it as being analogous to 'type I error' but it is really quite different. As we have mentioned, a major strength of the evidential paradigm lies in keeping the measure of errors separate from the measure 
of evidence $[6,7]$. The fundamental distinction is that $M_{0}$ is a measure of uncertainty, not of evidence. Consider a study that yields an $M_{0}$ value of 0.01 . All that this value is telling us is that if the null hypothesis is true - i.e. if the disease/trait really is not linked to these markers - there is only a 1 in 100 probability that we will observe a high LR that will mislead us into concluding linkage. This $M_{0}=0.01$ does not say anything about how strong the evidence is in favor of linkage; for that, we look at the actual value of the lod (mod) calculated from the given data set.

Future work will determine whether the asymptotic upper bound on the probability of misleading evidence [5] holds for MP mod scores, avoiding the requirement of calculating $M_{0}$ to ensure it is small.

\section{Appendix: Relative Magnitudes of Evidential Errors for Lods and Mods (Table 1)}

\section{Notation and Setup}

Consider a set of $m$ LRs, $L R_{i}, i=1, \ldots, m$, and let $L R_{\max }$ denote the maximum of the $m L R$ s. Any individual $L R_{i}$ corresponds to an individual lod analysis, whereas $L R_{\max }$ corresponds to a mod analysis.

Also consider two positive numbers: $0<a<b$.

- For each $L R_{i}$, define $P_{i} \equiv \operatorname{Pr}\left[L R_{i} \leq a\right], R_{i} \equiv \operatorname{Pr}\left[L R_{i} \geq b\right]$, and $Q_{i} \equiv \operatorname{Pr}\left[a<L R_{i}<b\right]=1-P_{i}-R_{i}$.

- For $L R_{\max }$, let $P_{*}$ be the probability that $L R_{\max }$ is less than or equal to $a$, i.e. $P_{*} \equiv \operatorname{Pr}\left[L R_{\max } \leq a\right]$, and similarly, $R_{*} \equiv$ $\operatorname{Pr}\left[L R_{\text {max }} \geq b\right]$ and $Q_{*} \equiv \operatorname{Pr}\left[a<L R_{\text {max }}<b\right]=1-P_{*}-R_{*}$.

First we examine $M$ and $S$; then we will turn to $W$.

\section{Implications for $M$ and $S$}

From basic probability principles, it is straightforward to show that the probability of the max LR being less than or equal to $a$ cannot exceed the probability for any of the individual LRs to be less than or equal to $a$. That is,

$$
\begin{aligned}
& \operatorname{Pr}\left[L R_{\max } \leq a\right]=\operatorname{Pr}\left[\bigcap_{i}\left(L R_{i} \leq a\right)\right] \leq \operatorname{Pr}\left[L R_{i} \leq a\right] \forall i, \text { i.e. } \\
& P_{*} \leq P_{i} \forall i .
\end{aligned}
$$

On the other hand, the probability of the max LR being greater than or equal to $b$ cannot be less than the probability for any of the individual LRs to be greater than or equal to $b$, i.e.

$$
\begin{aligned}
& \operatorname{Pr}\left[L R_{\text {max }} \geq b\right]=\operatorname{Pr}\left[\bigcup_{i}\left(L R_{i} \geq b\right)\right] \geq \operatorname{Pr}\left[L R_{i} \geq b\right] \forall i, \text { i.e. } \\
& R * \leq R_{i} \forall i .
\end{aligned}
$$

Applying these two results to the evidentialist paradigm, let $b$ represent $k$, our upper cutoff value for strong evidence supporting $H_{1}$, and let $a$ represent $1 / k$, our lower cutoff value for strong evidence supporting $H_{0}$.

- When $H_{0}$ is true, then $L R_{i}$ being less than or equal to $a$ represents strong evidence, whereas $L R_{i}$ being greater than or equal to $b$ represents misleading evidence; hence, $S_{i}=P_{i}$ and $M_{i}=R_{i}$; also $S_{*}=P_{*}$ and $M_{*}=R_{*}$. It follows from (A.1) and (A.2) that the probability of strong evidence will be less for mods than for lods, and the probability of misleading evidence will be greater for mods than for lods, as summarized in table 1 , in the ' $H_{0}$ true' column.

- When $H_{1}$ is true, it is the other way around: $L R_{i}$ being less than or equal to $a$ represents misleading evidence, whereas $L R_{i}$ being greater than or equal to $b$ represents strong evidence; hence, $M_{i}=P_{i}$ and $S_{i}=R_{i}$; and $M_{*}=P_{*}$ and $S_{*}=R_{*}$. Now the probability of strong evidence is greater, and the probability of misleading evidence less for mods than for lods, as in the ' $H_{1}$ true' column in table 1 .

These conclusions simply formalize the obvious fact that if one maximizes the lod over multiple models, one will achieve a LR that is greater than or equal to the LR resulting from only a single lod. If the null hypothesis is true, this procedure makes a misleading result more likely, whereas if the alternative is true, the procedure makes a not-misleading result more likely.

\section{Implications for $W$}

Under $H_{0}, W_{*}$ is generally greater for mods than for lods, whereas under $H_{1}, W_{*}$ is less for mods than for lods. We do not have definitive proofs of these relationships, but we show reasonable qualitative arguments here:

Working again with the $P, Q$, and $R$, and writing ' $L R$ between' as shorthand for the event $a<L R<b$ :

$$
Q_{*} \equiv \operatorname{Pr}\left[L R_{\max } \text { between }\right]=\operatorname{Pr}\left[L R_{i} \text { between }\right]-A_{i}+B_{i}, \text { for any } i,
$$

i.e.

$$
Q_{*}=Q_{i}-A_{i}+B_{i} \text {, for any } i,
$$

where $A_{i}$ and $B_{i}$ are defined as:

$$
\begin{aligned}
& A_{i} \equiv \operatorname{PR}\left[\left(L R_{i} \text { between }\right) \cap\left\{\bigcup_{j \neq i}\left(L R_{j} \geq b\right)\right\}\right] \\
& B_{i} \equiv \operatorname{PR}\left[\left(L R_{i} \leq a\right) \cap\left(\max _{j \neq i} L R_{i} \text { between }\right)\right]
\end{aligned}
$$

i.e. the probability that the $\max L R$ is 'between' $(Q *)$ can be expressed as -

- the probability that any one $L R$ - say, $L R_{i}$ - is 'between' $\left(Q_{i}\right)$

- minus the probability of the event in which $L R_{i}$ is 'between' but at least one other $L R_{j}$ is greater than or equal to $b\left(A_{i}\right)$

- plus the probability that $L R_{i}$ is less than or equal to $a$, and the maximum of all the other $L R_{j}$ is 'between' $\left(B_{i}\right)$.

Considering what $A_{i}$ and $B_{i}$ in (A.4) represent, we argue as follows (again letting $b$ represent $k$, and $a$ represent $1 / k$ ):

- In a well-designed study, when $H_{0}$ is true, all the $R_{i}$, which represent probabilities of misleading evidence for the individual LRs, will be very small. Then assuming a reasonable sample size, the $P_{i}$ (probabilities of strong evidence when $H_{0}$ is true) will be reasonably large, and the $Q_{i}$, which represent probabilities of weak evidence, will be reasonably small, or in any case not larger than the $P_{i}$. Each $A_{i}$ in (A.4) is largely governed by the presumably small probability that at least one of the $L R$ is greater than or equal to $b$, whereas $B_{i}$ represents the probability of the intersection of two events, neither of which has a very small probability. Thus, most often $B_{i}$ will be greater than $A_{i}$, and consequently $Q_{*}$ in (A.3) will be greater than any of the 
individual $Q_{i}$, or, equivalently, $W_{*}>W_{i} \vee i$, as summarized in table 1 , in the ' $H_{0}$ true' column.

- When $H_{1}$ is true, it is the other way around: Now in a welldesigned study, the $P_{i}$ represent probabilities of misleading evidence and will be very small. Each $A_{i}$ is governed by the probability that at least one of the $L R$ is greater than or equal to $b$, whereas $B_{i}$ cannot be bigger than $P_{i}$ for any LR, which should be very small. Most often $A_{i}$ will be greater than $B_{i}$, so that $Q_{*}$ in (A.3) will be less than any of the individual $Q_{i}$, or, equivalently, $W_{*}<W_{i} \forall i$, as in the ' $H_{1}$ true' column in table 1 . These approximate arguments concerning the $W$ are borne out by the results from our simulations under $H_{0}$ and $H_{1}$ (tables 1 and 5).

\section{Acknowledgments}

This work was supported in part by grants $\mathrm{MH}-48858$ from the National Institute of Mental Health (S.E.H.) and HG004314 from the Human Genome Research Institute (L.J.S.); also by the Natural Sciences and Engineering Research Council of Canada and the Ontario Ministry of Research and Innovation Early Researcher Award program (L.J.S.).

\section{References}

1 Vieland VJ: Bayesian linkage analysis, or: how I learned to stop worrying and love the posterior probability of linkage. Am J Hum Genet 1998;63:947-954.

2 Devlin B, Roeder K, Wasserman L: False discovery or missed discovery? Heredity 2003; 91:537-538.

3 Xing C, Elston RC: Distribution and magnitude of type I error of model-based multipoint lod scores: implications for multipoint mod scores. Genet Epidemiol 2006;30:447458.

- 4 Hodge SE, Rodriguez-Murillo L, Strug LJ, Greenberg DA: Multipoint lods provide reliable linkage evidence despite unknown limiting distribution: type I error probabilities decrease with sample size for multipoint lods and mods. Genet Epidemiol 2008;32:800815.

5 Royall R: On the probability of observing misleading statistical evidence. J Am Stat Assoc 2000;95:760-780.
6 Royall R: Statistical Evidence: A Likelihood Paradigm. London, Chapman \& Hall, 1997.

7 Blume JD: Likelihood methods for measuring statistical evidence. Stat Med 2002;21: 2563-2599.

8 Choi L, Caffo B, Rohde C: A survey of the likelihood approach to bioequivalence trials. Stat Med 2008;27:4874-4894.

-9 Zhang Z: Interpreting statistical evidence with empirical likelihood functions. Biom J 2009;51:710-720.

10 Hoch JS, Blume JD: Measuring and illustrating statistical evidence in a cost-effectiveness analysis. J Health Econ 2007;27:476495.

11 Blume JD, Su L, Olveda RM, McGarvey ST: Statistical evidence for GLM regression parameters: a robust likelihood approach. Stat Med 2007;26:2919-2936.

12 Strug LJ, Hodge SE: An alternative foundation for the planning and evaluation of linkage analysis. I. Decoupling 'error probabilities' from 'measures of evidence'. Hum Hered 2006;61:166-188.
3 Strug LJ, Rohde CA, Corey PN: An introduction to evidential sample size calculations. Am Stat 2007;61:207-212.

14 Strug LJ, Hodge SE, Chiang T, Pal DK, Corey PN, Rohde C: A pure likelihood approach to the analysis of genetic association data: an alternative to Bayesian and Frequentist analysis. Eur J Hum Genet 2010;18:933-941.

15 Strug LJ, Hodge SE: An alternative foundation for the planning and evaluation of linkage analysis. II. Implications for multiple test adjustments. Hum Hered 2006;61:200-209.

16 Edwards AWF: Likelihood, expanded ed. Baltimore, Johns Hopkins University Press, 1992.

17 Pawitan Y: In All Likelihood: Statistical Modelling and Inference Using Likelihood. New York, Oxford University Press, 2001.

18 Kalbfleisch JD: Comment on Royall's 'On the probability of observing misleading statistical evidence'. J Am Stat Assoc 2000;95: 770-771. 